\title{
New mould sealer cures at room temperature
}

enkel has a new mould sealer in its product portfolio. The
sealer, called Loctite Frekote CS-122, was developed as a standard sealer for low- to medium-gloss composite materials,

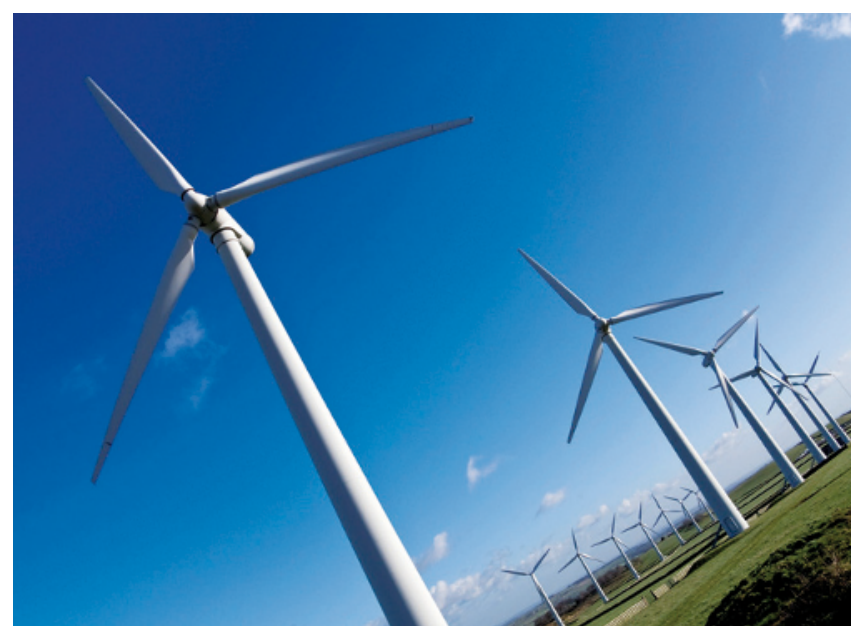

but it can also be applied to both metal and epoxy moulds and is compatible with Frekote release agents.

When applied to a mould, the sealer forms micro-thin, thermally stable surfaces that are chemically bonded to the mould surface, thus sealing micro porosity. Compared to the predecessor product, curing time is reduced to two hours. The new product can also seal larger porosities because it allows thicker coatings to be applied. The sealer can be either sprayed or wiped on the surface at temperatures of between $13{ }^{\circ} \mathrm{C}$ and $40{ }^{\circ} \mathrm{C}$. Energy-consuming preheating of the mould is therefore unnecessary. After curing, the sealing remains stable at temperatures as high as $400{ }^{\circ} \mathrm{C}$.

For more information, please contact: Henkel AG \& Co. KGaA, www.henkel.com

A new product on the market is a sealer for low- to medium-gloss composite materials such as rotor blades.

\section{Particle size measurement for dry powders}

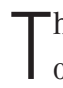

he dry dispersion of powders by means

of laser diffraction is seen as a fast and ecologically neutral method of particle size analysis. However, until now the use of this method has been somewhat limited in the case of fragile, brittle particles. The limiting factor was the need to introduce sufficient energy for dispersion but without destroying the fragile particles. This problem is now remedied by the $\mathrm{Ae}^{-}$ ro-S dry powder disperser developed by Malvern, which allows the equally new Mastersizer 3000 particle size analyser to analyse the particle size of all kinds of dry power samples and enables users to measure materials such as cement, coffee, cohesive minerals and even fragile pharmaceutical materials with a size range of $0.1 \mu \mathrm{m}$ to $3.5 \mathrm{~mm}$ in a dry state. Due to its fast and efficient analysis with an extremely high data acquisition rate, the new disperser provides stable, accurate dry measurements of even the most demanding samples with very wide particle size distributions.
The new dry disperser is a completely new development. A modular venturi dispersing system enables the user to select the most suitable dispersion mechanism for the sample being analysed. The venturi nozzle that is included as standard equipment is suitable for a very broad spectrum of samples and also disperses cohesive materials while maintaining the integrity of the fragile particles. An optional venturi that additionally disperses the sample by direct impaction is available for more demanding requirements such as breaking up agglomerated samples and the dispersion of very cohesive materials. The dispersion system also features sensitive dispersion pressure control to within $+/-0.1$ bar over the range from 0.1 to 4 bar, which allows precise adaptation of the energy input according to the sample. Sample trays in different sizes can be selected according to the size of the sample. The enclosed measuring cell delivers bias-free measurements while minimising user exposure to the sample. All steps of the analysis - feeding the sample, dispersing, measu-

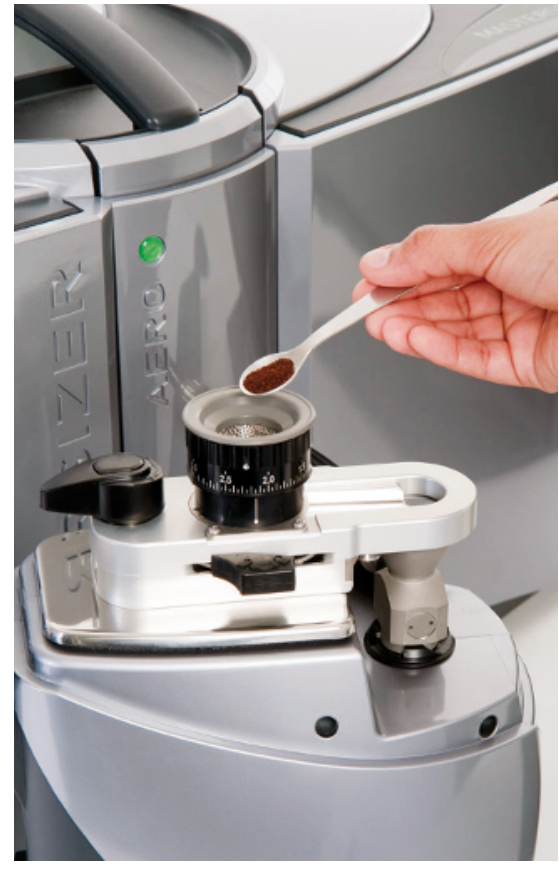

ring and cleaning - are completely controlled by the software via the System Manager interface.

For more information, please contact: Malvern Instruments GmbH, D-71083 Herrenberg, www.malvern.de/MS3000 